\title{
TERAPIA FOTODINÁMICA CON VERTEPORFINA EN NEOVASCULARIZACIÓN COROIDEA DESPUÉS DE CIRUGÍA REFRACTIVA
}

\section{PHOTODYNAMIC THERAPY WITH VERTEPORFIN IN CHOROIDAL NEOVASCULARIZATION AFTER REFRACTIVE SURGERY}

\begin{abstract}
AMAT-PERAL P ${ }^{1}$, LUGO F ${ }^{1}$, MONTERO JA², DE-LA-VEGA C ${ }^{3}$, ARÉVALO JF${ }^{4}$, ALIÓ Y SANZ JL $^{5}$
\end{abstract}
\section{RESUMEN}

Propósito: Analizar los resultados obtenidos usando TFD (terapia fotodinámica) con verteporfina como primera elección para tratar la NVC (neovascularización coroidea) subfoveal y yuxtafoveal en pacientes con miopía magna corregida mediante cirugía refractiva (laser-assisted in situ keratomileusis -LASIK- y lentes fáquicas de cámara anterior - LFCA-).

Método: Se estudiaron catorce ojos que presentaban alta miopía a los que se les había realizado LASIK (siete casos) o implante de lente intraocular -LIO- (siete casos), que desarrollaron NVC y fueron tratados con TFD con verteporfína.

Resultados: La media Mejor Agudeza Visual Corregida (MAVC) después de la cirugía fue 0,45 (DE: 0,17 ; rango: 0.2 a 0.8 ), con equivalente esférico residual (EER) -0,5 (DE: 1,8D; rango: 1 a 5,5D). La MAVC después de NVC fue 0,10 (DE: 0,19; rango: 0,025 a 0,7$)$. NVC fue tratada con TFD (media de 2,0 DE: 0,8 tratamientos). La media MAVC mejoró hasta 0,22 (DE: 0,18; rango: 0;1 a 0,63) (EER -1,4 DE: 1,4D; rango: 0,5 a -4D).

\section{ABSTRACT}

Purpose: To analyze the results obtained with Photodynamic Therapy (PDT) to treat subfoveal and juxtafoveal Choroidal Neovascularization $(\mathrm{CNV})$ in patients with high myopia corrected by LaserAssisted in situ Keratomileusis (LASIK) or by implanting a Phakic Intraocular Lens (PIOL).

Methods: We analyzed the results from 14 highly myopic eyes corrected by LASIK (seven cases) or by PIOL implantation (seven cases), which later developed CNV and were treated by PDT with verteporfin.

Results: Mean Best Corrected Visual Acuity (BCVA) after refractive surgery was 0.45 SD 0.17 (range, 0.2 to 0.8 ), with residual spherical equivalent (RSE) -0.5 SD 1.8 D (range, 1 to 5.5 D). After CNV appearance, BCVA was 0.10 SD 0.19 (range, 0.025 to 0.7$)$. CNV was treated in all cases by PDT (mean, 2.0 SD 0.8 treatments). After CNV closure, the mean BCVA improved up to 0.22 SD 0.18 (range, 0.1 to 0.63 ) (RSE - 1.4 SD 1.4 D, range, 0.5 to -4 D). Differences in RSE after refractive surgery

\footnotetext{
Recibido: 27/2/07. Aceptado: 25/8/08 Hernández. Alicante. España.

Correspondencia:

Pedro Amat-Peral

C/. Calderón de la Barca, 29, 2. ${ }^{\circ}$ A

03004 Alicante

España

E-mail: pamatp@yahoo.es
}

Instituto Oftalmológico de Alicante-Vissum. Alicante. España. Clínica Oftalmológica Centro Caracas. Caracas. Valenzuela.

1 Licenciado en Medicina. Instituto Oftalmológico de Alicante. Unidad de Retina y Vítreo. Alicante. España.

2 Doctor en Medicina. Departamento de Oftalmología. Universidad Miguel Hernández. Alicante. España.

3 Doctora en Medicina. Instituto Oftalmológico de Alicante. Unidad de Retina y Vítreo. Departamento de Oftalmología. Universidad Miguel

4 Doctor en Medicina. Clínica Oftalmológica Centro Caracas. Servicio de Retina y Vítreo. Caracas. Valenzuela.

5 Doctor en Medicina. Instituto Oftalmológico de Alicante. Unidad de Cirugía Refractiva. Alicante. España.

Comunicación presentada en el LXXXII Congreso de la S.E.O. (A Coruña 2006). 
Las diferencias en EER después de cirugía refractiva y después de TFD, y las diferencias en MAVC antes y después de TFD no fueron estadísticamente significativas $(p=0,82$ y $p=0,06$, respectivamente, aplicada t de Student para datos apareados).

Conclusiones: El empleo de TFD para el tratamiento de la NVC tras la cirugía refractiva permite mantener la MAVC sin inducir cambios en el equivalente esférico.

Palabras clave: Cirugía refractiva, neovascularización coroidea, terapia fotodinámica, LASIK, lentes fáquicas de implantación intraocular, miopía patológica, equivalente esférico residual. and after PDT, and differences between BCVA after CNV appearance and final were not statistically significant $(\mathrm{p}=0.82$ and $\mathrm{p}=0.06$, respectively, Student's t test paired data).

Conclusion: We consider that PDT is effective in achieving closure of CNV in myopic patients after refractive surgery without inducing changes in spherical equivalent (Arch Soc Esp Oftalmol 2008; 83: 539-544).

Key words: Refractive surgery, choroidal neovascularization, photodynamic therapy, LASIK, phakic intraocular lens implantation, pathologic myopia, residual spherical equivalent.

\section{INTRODUCCIÓN}

La Neovascularización Coroidea (NVC) es una complicación poco frecuente pero importante en ojos con Miopía Magna (MM) (1-4) que puede aparecer después de la cirugía refractiva. La aparición de NVC ha sido descrita después de queratectomía fotorrefractiva (5), después de laser-assisted in situ keratomileusis (LASIK) (6) y después de lentes fáquicas de cámara anterior (LFCA) (7).

Hasta la aparición de la terapia antiangiogénica y sus buenos resultados en estudios preliminares (811), el tratamiento de la NVC extrafoveal era la fotocoagulación con láser térmico. La translocación macular (12-14) y la terapia fotodinámica (TFD) con verteporfina se reservaban para el tratamiento de las NVC subfoveales (15-17) y de las yuxtafoveales (7) en ojos miopes.

El objetivo de este artículo es analizar los resultados obtenidos usando TFD con verteporfina como primera elección para tratar la NVC subfoveal y yuxtafoveal en pacientes con MM corregida mediante cirugía refractiva (LASIK y LFCA).

\section{SUJETOS, MATERIAL Y MÉTODOS}

Catorce pacientes con MM (14 ojos) corregidos con cirugía refractiva que habían desarrollado NVC subfoveal y yuxtafoveal fueron examinados en el Servicio de Retina y Vítreo del Instituto Oftalmológico de Alicante (12 pacientes, LASIK o LFCA) y en la Clínica Oftalmológica Centro de Caracas (dos pacientes, LASIK) e incluidos para estudio.

\section{Criterios de Inclusión}

- Error refractivo mayor de -6 dioptrías antes de cirugía refractiva y/o longitud axial mayor de $26 \mathrm{~mm}$.

- Cirugía refractiva previa (LASIK o LFCA) para corregir alta miopía.

- Ningún tratamiento láser previo sobre la mácula.

- Evidencia clínica de NVC subfoveal o yuxtafoveal confirmada con angiografía fluoresceína.

- Consentimiento informado por escrito.

\section{Criterios de exclusión}

- Signos clínicos de Degeneración Macular Asociada a la Edad (DMAE), como drusas blandas, Síndrome de Presunta Histoplasmosis Ocular (SPHO), así como otras patologías que puedan desarrollar NVC en su evolución.

- Embarazo o lactancia.

- Pacientes que no son aptos para TFD.

La media de edad fue de 36,5 (DE: 10,6 años; rango: 24 a 52 años). Diez pacientes fueron mujeres y cuatro hombres. De los ojos que se trataron, ocho fueron derechos y seis izquierdos. El seguimiento medio tras cirugía refractiva fue de 46,1 (DE: 27,2 meses; rango: 9 a 112 meses). Los datos de estos pacientes están descritos en las tablas I y II.

La TFD con verteporfin (Visudyne ${ }^{\circledR}$, Novartis AG, Bülach, Suiza) fue desarrollada como se describe previamente $(16,17)$. Los pacientes recibieron tratamientos con TFD sólo si la NVC había sido demostrada con angiografía fluoresceínica (AGF), y se realizaron en el periodo entre una semana después de la última AGF con un intervalo de tres meses entre cada sesión. 
Criterios de exclusión

- Error refractivo mayor de - 6 dioptrías antes de la cirugía refractiva y/o un eje axial mayor de $26 \mathrm{~mm}$.

- Cirugía refractiva previa (LASIK o LFCA) para corregir alta miopía (patológica).

- Ninguna otra patología ocular diferente a aquellas relacionadas con la alta miopía.

- Ningún tratamiento previo con láser sobre la mácula.

- Evidencia clínica de NVC subfoveal o yuxtafoveal, confirmada con angiografía fluoresceínica (AGF).

- Consentimiento informado por escrito.

El seguimiento medio después de la primera TFD fue de 13,2 (DE: 7;7 meses; rango: de 5 a 30 meses).

La $\mathrm{t}$ de Student para datos pareados fue usada para comparar las diferencias entre la MAVC y el equivalente esférico residual.

\section{RESULTADOS}

La MAVC media después de la cirugía fue de 0,45 (DE: 0,17; rango: 0,2 a 0,8), con equivalente esférico residual medio de $-1,5$ (DE: 1,8D; rango: 1 a 5,5D). El tiempo medio entre la cirugía refractiva y la aparición de NVC fue de 33 (DE: 24 meses; rango: 1 a 87). La NVC fue subfoveal en once casos y yuxtafoveal en tres casos (tabla I).
- Todos los pacientes con signos clínicos de Degeneración Macular Asociada a la Edad (DMAE), como drusas, Síndrome de Presunta Histoplasmosis Ocular (SPHO), así como otras patologías que puedan desarrollar NVC en su evolución.
La NVC apareció en siete casos después de la implantación de LFCA. En cinco casos fueron implantadas lentes fáquicas de soporte angular (casos n. ${ }^{\circ} 1$ a 5), un caso con lentes fáquicas fijadas en iris (caso n. ${ }^{\circ}$ 7) y un caso (caso n. ${ }^{\circ}$ 6) con lente fáquica de cámara posterior (PRL). La NVC apareció en siete casos después de LASIK (casos n. ${ }^{\circ} 8$ a 14).

La MAVC fue 0,10 (DE: 0,19; rango: 0,025 a $0,7)$ después de NVC (tabla II). Las diferencias entre la MAVC después de la cirugía refractiva y la aparición de NVC fueron estadísticamente significativas ( $\mathrm{p}=0.01, \mathrm{t}$ de Student para datos pareados).

Después del cierre de la NVC (tres meses después de la última TFD, en cada caso) la MAVC media mejoró hasta 0,22 (DE: 0,18; rango: 0,1 a 0,63 ). Estos cambios no fueron estadísticamente significa-

\section{Tabla II.}

\begin{tabular}{|c|c|c|c|c|c|c|c|c|c|c|c|c|c|}
\hline Paciente & Edad & Sexo & $\begin{array}{c}\mathrm{EE} \\
\text { Inicial }\end{array}$ & $\mathrm{EEr}$ & $\begin{array}{l}\text { Patología } \\
\text { retiniana }\end{array}$ & $\begin{array}{c}\text { Periodo } \\
\text { de estudio }\end{array}$ & $\begin{array}{l}\text { Cirugía } \\
\text { Refractiva }\end{array}$ & $\begin{array}{c}\text { MAVC } \\
\text { NVC }\end{array}$ & Tto. & $\begin{array}{c}\text { EE } \\
\text { Final }\end{array}$ & $\begin{array}{l}\text { MAVC } \\
\text { Final }\end{array}$ & $\begin{array}{c}\text { Seguimiento } \\
\text { Total }\end{array}$ & $\begin{array}{c}\text { Seguimiento } \\
\text { Retina }\end{array}$ \\
\hline 1 & 44 & $\mathrm{H}$ & $-16,25$ & -2 & NVC Subf & 18 & LFCA & 0,16 & $\mathrm{TFD}(2)$ & -1 & 0,25 & 48 & 30 \\
\hline 2 & 30 & M & -22 & -1 & NVC Subf & 87 & LFCA & 0,50 & TFD (2) & - & 0,50 & 112 & 25 \\
\hline 3 & 30 & $\mathrm{H}$ & $-19,25$ & -1 & NVC Yuxtaf & 45 & LFCA & 0,10 & TFD (2) & $-2,50$ & 0,50 & 50 & 5 \\
\hline 4 & 28 & M & $-22,5$ & +1 & NVC Yuxtaf & 3 & LFCA & 0,40 & TFD (2) & - & 0,50 & 12 & 9 \\
\hline 5 & 25 & M & $-26,75$ & $+0,75$ & NVC Subf & 24 & LFCA & 0,70 & TFD (2) & - & 0,63 & 39 & 15 \\
\hline 6 & 35 & $\mathrm{H}$ & $-8 *$ & -1 & NVC Yuxtaf & 1 & LFCA & 0,20 & TFD (1) & $+0,50$ & 0,50 & 9 & 8 \\
\hline 7 & 24 & $\mathrm{H}$ & -23 & $-0,75$ & NVC Subf & 5 & LFCA $* *$ & 0,025 & TFD (1) & $-0,75$ & 0,20 & 11 & 6 \\
\hline 8 & 38 & M & $-16,25$ & -1 & NVC Subf & 60 & LASIK & 0,10 & TFD (1) & $-3,5$ & 0,10 & 76 & 16 \\
\hline 9 & 27 & M & $-15,75$ & $-3,25$ & NVC Subf & 49 & LASIK & 0,20 & TFD (1) & -3 & 0,50 & 66 & 17 \\
\hline 10 & 47 & M & -8 & -1 & NVC Subf & 46 & LASIK & 0,13 & TFD (2) & -2 & 0,20 & 54 & 8 \\
\hline 11 & 28 & M & $-13,5$ & - & NVC Subf & 37 & LASIK & 0,05 & TFD (4) & - & 0,10 & 55 & 20 \\
\hline 12 & 52 & M & -13 & -4 & NVC Subf & 31 & LASIK & 0,16 & TFD (3) & -4 & 0,20 & 39 & 8 \\
\hline 13 & 52 & M & -8 & $-2,75$ & NVC Subf & 41 & LASIK & 0,25 & TFD (2) & $-1,75$ & 0,16 & 46 & 5 \\
\hline 14 & 51 & M & -13 & $-5,5$ & NVC Subf & 16 & LASIK & 0,06 & TFD (3) & $-2,25$ & 0,13 & 34 & 18 \\
\hline Media & 36,5 & & -16 & $-1,5$ & & 33 & & 0,10 & 2 & $-1,4$ & 0,22 & 46,1 & 13,2 \\
\hline $\mathrm{DE}$ & 10,6 & & 6 & 1,8 & & 24 & & 0,19 & 0,8 & 1,4 & 0,18 & 27,2 & 7,7 \\
\hline Rango & $24 / 52$ & & $-8 /-26,75$ & $1 /-5,5$ & & $1 / 87$ & & $0,70 / 0,025$ & $1 / 4$ & $0,5 /-4$ & $0,63 / 0,10$ & $9 / 112$ & $5 / 30$ \\
\hline
\end{tabular}

NVC: Neovascularización coroidea; TFD: Terapia fotodinámica; LFCA: Lentes fáquicas de cámara anterior; LASIK: Laser assisted in situ keratomileusis; EE: Equivalente esférico; r: después de la cirugía refractiva; MAVC: Mejor Agudeza Visual Corregida; H: Hombre; M: Mujer; DS: Desviación estandar; Subf: Subfoveal; Yuxtaf: Yuxtafoveal; Seguimiento: en meses. * Después de LASIK. ** fijación y reposición nasal. 
tivos, aunque están muy próximos a ser significativos ( $\mathrm{p}=0,06, \mathrm{t}$ de Student para datos pareados).

Cuatro ojos $(28,5 \%)$ mejoraron más de cuatro líneas, cuatro ojos $(28,5 \%)$ dos o tres líneas y cinco ojos (42\%) no mostraron cambios (una línea o ninguna línea, ganada o perdida). Sólo un ojo $(7,1 \%)$ perdió dos líneas.

Se necesitaron de una a cuatro sesiones (una sesión en cuatro casos y cuatro sesiones en un caso) con TFD para el cierre de la NVC (media: 2,0; DE: 0,8 tratamientos).

El equivalente esférico residual después del tratamiento con TFD fue de $-1,4$ (DE: 1,4D; rango: 0,5 a 4D). Las diferencias entre el equivalente esférico residual después de TFD y después de cirugía refractiva no fueron estadísticamente significativas $(\mathrm{p}=0,82$, $\mathrm{t}$ de Student para datos pareados).

\section{DISCUSIÓN}

La NVC es uno de los mayores cambios de la miopía degenerativa y está asociada con las estrías lacas (2), que se encontraron en $82 \%$ de los ojos con NVC (1). Cuando la rotura de la membrana de Bruch se produce, permite la progresión de la NVC (1). La incidencia de NVC en alta miopía se produce entre un 4 a un $11 \%$ (3). La miopía mayor de -6D se ha relacionado en la formación de NVC (4), y la alta miopía está considerada la principal causa de NVC (62\%) en un estudio en pacientes jóvenes (4).

Existen publicaciones que demuestran la incidencia de la NVC en ojos con MM después de la cirugía refractiva. Sólo se ha publicado un caso de 5.936 ojos miopes intervenidos mediante PRK (5), en el $0,10 \%$ de 2.955 ojos miopes corregidos con LASIK (6) y en 1,70\% de 294 ojos miopes corregidos mediante LFCA de soporte angular (7). Solamente se ha documentado un caso después de LASIK en pacientes hipermétropes (18).

El propósito de nuestro estudio es investigar la eficacia y seguridad de la TFD con verteporfina en el tratamiento de la NVC subfoveal y yuxtafoveal en ojos miopes después de la cirugía refractiva.

Desde que la extracción de la NVC se abandonó a causa de sus resultados irregulares (19), se consideraron diferentes soluciones cuando aparecía la NVC subfoveal. Nosotros podríamos dejar sin tratamiento el paciente y ver el curso espontáneo de la enfermedad. La historia natural de la NVC en ojos MM después de tres años es peor que después de TFD, aun- que puede ser mejor en pacientes por debajo de los 40 años que en pacientes por encima de 40 años (12). Otros autores habían demostrado previamente la mala agudeza visual asociada a la evolución espontánea de la NVC en ojos MM $(1,20,21)$.

También se han publicado buenos resultados después de la translocación macular. Fujikado publicó una mejoría de la MAVC en ocho de 11 pacientes y un empeoramiento en sólo un caso a los que se les aplicó una translocación macular con retinotomía $360^{\circ}$ (13); desprendimiento de retina en dos casos (1 con vitreorretinopatía proliferativa) hemorragia de vítreo persistente y un agujero macular en un ojo después de este procedimiento.

La translocación macular limitada es un procedimiento quirúrgico más simple pero han sido publicadas varias complicaciones por Fujii et al (22) en al menos un $34,6 \%$. Otro factor a tener en cuenta es la inducción de alto astigmatismo con acortamiento escleral en estos pacientes (12), especialmente desde que habían sido tratados mediante cirugía refractiva. Glacet-Bernard et al. demostraron buenos resultados en un $67 \%$ y sin cambios en un $33 \%$ de sus pacientes, pero también publicaron desprendimientos de retina, neovascularización en el sitio de inyección y recurrencia de la NVC.

VIP 1 mostró que la TFD con verteporfina puede lograr un mejor índice de estabilidad o mejoría en la MAVC en pacientes MM con NVC (15). En nuestra experiencia de TFD con verteporfina en pacientes con miopes magna se observan buenos resultados en términos de MAVC y cierre de la NVC, con mejora en $61 \%$ de los pacientes de 55 años o más jóvenes, y empeoramiento en el 28\%. En nuestros estudios no hemos encontrado ninguna complicación asociada a la TFD (16). Sólo la aparición de fibrosis subretiniana después de la TFD en NVC en miopes magnos puede ser subrayada, que no siempre significa una pérdida de la MAVC (17).

Nuestro estudio muestra el ejemplo de catorce ojos con NVC después de la corrección de miopía con LASIK o con implante de LFCA, tratados con TFD con un seguimiento medio de 46,1 (DE: 27,2 meses; rango: 9 a 112 meses). La MAVC mejoró después del tratamiento con TFD y el cierre de la NVC, desde 0,10 a 0,22 ( $p=0,06$, $t$ de Student para datos pareados). Se obtuvieron resultados similares en un artículo publicado por nosotros16 en el que la mejoría de la MAVC para pacientes por debajo de 55 años (edad media 44,9) estuvieron cerca de ser estadísticamente significativos ( $\mathrm{p}=0,07, \mathrm{t}$ de Student para datos apareados). 
En el presente estudio, ocho ojos $(57,1 \%)$ mostraron una mejoría en más de 2 líneas y cinco ojos $(35,7 \%)$ no mostraron cambios (una o ninguna línea, ganada o perdida). Un ojo $(7,1 \%)$ perdió más de una línea.

Otro factor importante a ser considerado en estos casos es el posible cambio en el equivalente esférico. Estos pacientes se sometieron a cirugía para corregir su defecto miópico. El equivalente esférico residual después del tratamiento con TFD (-1,4; DE: $1,4 \mathrm{D})$ en nuestro estudio no mostraba cambios ( $\mathrm{p}=0,82$, $\mathrm{t}$ de Student para datos pareados) comparados con equivalente esférico después de la cirugía refractiva $(1,5 ; \mathrm{DE}: 1,8 \mathrm{D})$.

El único resultado diferente en este estudio ha sido el bajo número de retratamientos (media 2,0) comparado a 2,6 de nuestro anterior estudio (16) y a 3,4 en el estudio VIP 1 (15). Estos dos estudios incluyeron por lo menos un año de seguimiento después del seguimiento después de TFD comparado a los 13,2; DE: 7,7 meses de estos pacientes.

Según nuestros resultados en términos de MAVC y la ausencia de complicaciones y cambios en el equivalente esférico residual, la TFD podría ser considerada como una aproximación terapéutica para NVC en pacientes miopes después de la cirugía refractiva, sin embargo, dados los buenos resultados publicados en estudios preliminares sobre el tratamiento con antiangiogénicos de la NVC en pacientes con MM pensamos que éste será el futuro del tratamiento de esta patología (8-11). Este hecho necesita ser confirmado en próximos estudios con más tiempo de seguimiento y mayor número de pacientes.

\section{BIBLIOGRAFÍA}

1. Hotchkiss ML, Fine SL. Pathologic myopia and choroidal neovascularization. Am J Ophthalmol 1981; 91: 177-183.

2. Johnson DA, Yannuzzi LA, Shakin JL, Lightman DA. Lacquer cracks following laser treatment of choroidal neovascularization in pathologic myopia. Retina 1998; 18 : 118-124.

3. Grossniklaus HE, Green WR. Pathologic findings in pathologic myopia. Retina 1992; 12: 127-133.

4. Cohen SY, Laroche A, Leguen Y, Soubrane G, Coscas GJ. Etiology of choroidal neovascularization in young patients. Ophthalmology 1996; 103: 1241-1244.

5. Ruiz-Moreno JM, Artola A, Ayala MJ, Claramonte P, Alio $J L$. Choroidal neovascularization in myopic eyes after photorefractive keratectomy. J Cataract Refract Surg 2000; 26: 1492-1495.

6. Ruiz-Moreno JM, Pérez-Santonja JJ, Alió JL. Choroidal neovascularization in myopic eyes after laser-assisted in situ keratomileusis. Retina 2001; 21: 115-120.
7. Ruiz-Moreno JM, de la Vega C, Ruiz-Moreno O, Alió JL. Choroidal neovascularization in phakic eyes with anterior chamber intraocular lenses to correct high myopia. J Cataract Refract Surg 2003; 29: 270-274.

8. Yamamoto I, Rogers AH, Reichel E, Yates PA, Duker JS. Intravitreal bevacizumab (Avastin) as treatment for subfoveal choroidal neovascularisation secondary to pathological myopia. Br J Ophthalmol 2007; 91: 157-160.

9. Sakaguchi H, Ikuno Y, Gomi F, Kamei M, Sawa M, Tsujikawa $M$, et al. Intravitreal injection of bevacizumab for choroidal neovascularisation associated with pathological myopia. Br J Ophthalmol 2007; 91: 161-165.

10. Rosenfeld PJ. Intravitreal Avastin for choroidal neovascularisation in pathological myopia: the controversy continues. Br J Ophthalmol 2007; 91: 128-130.

11. Ruiz-Moreno JM, Gomez-Ulla F, Montero JA, Ares S, Lopez-Lopez F, Rodríguez, $M$, et al. Intravitreous bevacizumab to treat subfoveal choroidal neovascularization in highly myopic eyes: short-term results. Eye 2007; doi:10.1038/sj.eye.6703052.

12. Fujikado T, Ohji M, Saito Y, Hayashi A, Tano Y. Visual function after foveal translocation with scleral shortening in patients with myopic neovascular maculopathy. Am J Ophthalmol 1998; 125: 647-656.

13. Fujikado T, Ohji M, Kusaka S, Hayashi A, Kamei M, Okada $A A$, et al. Visual function after foveal translocation with 360-degree retinotomy and simultaneous torsional muscle surgery in patients with myopic neovascular maculopathy. Am J Ophthalmol 2001; 131: 101-110.

14. Fujikado T, Ohji M, Hayashi A, Kusaka S, Tano Y. Anatomic and functional recovery of the fovea after foveal translocation surgery without large retinotomy and simultaneous excision of a neovascular membrane. Am J Ophthalmol 1998; 126: 839-842.

15. Verteporfin in Photodynamic Therapy Study Group. Photodynamic therapy of subfoveal choroidal neovascularization in pathologic myopia with verteporfin. 1-year results of a randomized clinical trial-VIP report $n^{\circ} .1$. Ophthalmology 2001; 108: 841-852.

16. Montero JA, Ruiz-Moreno JM. Verteporfin photodynamic therapy in highly myopic subfoveal choroidal neovascularisation. Br J Ophthamol 2003; 87: 173-176.

17. Ruiz-Moreno JM, Montero JA. Subretinal fibrosis after photodynamic therapy in subfoveal choroidal neovascularisation in highly myopic eyes. Br J Ophthamol 2003; 87: 856-859.

18. Arevalo JF, Ramirez E, Suarez E, Morales-Stopello J, Cortez $R$, Ramírez $G$, et al. Incidence of vitreoretinal pathologic conditions within 24 months after laser in situ keratomileusis. Ophthalmology 2000; 107: 258-262.

19. Ruiz-Moreno JM, de la Vega C. Surgical removal of subfoveal choroidal neovascularisation in highly myopic patients. Br J Ophthalmol 2001; 85: 1041-1043.

20. Avila MP, Weiter JJ, Jalkh AE, Trempe CL, Pruett RC, Schepens CL. Natural history of choroidal neovascularization in degenerative myopia. Ophthalmology 1984; 91 : 1573-1581.

21. Secretan M, Kuhn D, Soubrane G, Coscas G. Long-term visual outcome of choroidal neovascularization in pathologic myopia: natural history and laser treatment. Eur J Ophthalmol 1997; 7: 307-316.

22. Fujii GY, Pieramici DJ, Humayun MS, Schachat AP, Reynolds SM, Melia M, et al. Complications associated with 
limited macular translocation. Am J Ophthalmol 2000; 130: 751-762.

23. Glacet-Bernard A, Simon P, Hamelin N, Coscas G, Soubrane G. Translocation of the macula for manage- ment of subfoveal choroidal neovascularization: comparison of results in age-related macular degeneration and degenerative myopia. Am J Ophthalmol 2001; 131: 78-89. 